Prólogo

\section{DESCOLONIZACIÓN DE LA CIENCIA DE LOS DESASTRES: ENFOQUES DESDE LATINOAMÉRICA Y CARIBE}

\author{
Victor Marchezini ${ }^{{ }^{*}}$, Claudia González-Muzzio ${ }^{2,3}$ y Aracely Martínez Rodas 4
}

\section{RESUMEN}

Esta breve introducción para el número especial "Descolonización de la ciencia de los desastres: enfoques desde Latinoamérica y Caribe" (Vol. 5, Núm. 2) busca promover la pluralidad del conocimiento mediante la valoración de las ontologías y epistemologías locales para descolonizar los estudios de desastres y trascender a los enfoques, fuentes, conceptos, metodologías, valores y lenguajes occidentales 'bien establecidos' que son predominantemente ajenos a los puntos críticos de desastres y riesgos que afectan a la región. Algunos de los temas abordados en el número especial incluyen: pensamiento social latinoamericano ante los desastres y epidemias; descolonización de la ciencia de los desastres; cosmovisiones, conocimiento de los pueblos oprimidos y saberes alternativos en la investigación de desastres; ecología de los saberes; antropología de los desastres; sociología de los desastres; nuevas geopolíticas y biopolíticas en los desastres y pandemias; modelos de desarrollo y producción social de los riesgos de desastres; procesos de vulneración, formas de opresión, desigualdad y pobreza en los desastres; racismo estructural y ambiental en los desastres; desastres olvidados y pueblos oprimidos; capitalismo del desastre; dimensiones del poder en la producción social de los riegos y en los desastres; éticas de investigación de desastres; entre otros.

\section{PALABRAS CLAVES}

Descolonización, Ciencia de los desastres, Riesgo, Amenazas, Latinoamérica, El Caribe

\section{DECOLONIZATION OF DISASTER SCIENCE: APPROACHES FROM LATIN AMERICAAND THE CARIBBEAN}

\section{ABSTRACT}

This brief introduction to the special issue "Decolonization of disaster science: Approaches from Latin America and the Caribbean" (Vol. 5, No. 2) seeks to promote the plurality of knowledge by assessing local ontologies and epistemologies. Ultimately, this look at how decolonize studies and how transcend 'well-established' Western approaches, sources, concepts, methodologies, values and even languages, which are predominantly foreign views to the disaster and risk hotspots affecting the region. Some of the topics addressed in the special issue include: Latin American social thought in the face of disasters and epidemics; decolonization of the science of disasters; worldviews, knowledge of oppressed peoples and alternative knowledge in disaster research; ecology of knowledge; anthropology of disasters; sociology of disasters; new geopolitics and biopolitics in disasters and pandemics; development models and social production of disaster risks; processes of violation, forms of oppression, inequality and poverty in disasters; structural and environmental racism in disasters; 'invisible' disasters and oppressed peoples; disaster capitalism; dimensions of power in the social production of risks and disasters; disaster ethics; among others.

\section{KEYWORDS}

Decolonization, Disaster science, Risk, Hazards, Latin America, The Caribbean
1. Centro Nacional de

Monitoramento e Alertas de Desastres Naturais CEMADEN, São Paulo, Brasil.

2. Ámbito Consultores, Santiago, Chile.

3. GRID Chile, Santiago, Chile.

4. Maestría en Desarrollo, Universidad del Valle de Guatemala, Ciudad de Guatemala, Guatemala.

*Autor de correspondencia: victor.marchezini@cemaden. gov.br

Identificador:

http://revistareder.com/

handle-0719-8477-2021-120

\section{RECIBIDO}

5 de junio de 2021

PUBLICADO

1 de julio de 2021

Formato cita

Recomendada (APA):

Marchezini, V.,

González-Muzzio, C.

\& Martínez Rodas, A.

(2021). Descolonización

de la ciencia de los desastres: enfoques desde Latinoamérica y Caribe. Revista de Estudios

Latinoamericanos sobre Reducción del Riesgo de Desastres REDER, 5(2), 1-4. http://revistareder.com/ handle-0719-8477-2021-120

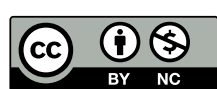

Todos los artículos publicados en REDER siguen una política de Acceso Abierto y se respaldan en una Licencia CreativeCommons Atribución-NoComercial 4.0 Internacional.

Revista de Estudios Latinoamericanos sobre Reducción del Riesgo de Desastres (REDER)

Diseño: Lupe Bezzina 


\section{PRÓLOGO}

El número especial Descolonización de la ciencia de los desastres: enfoques desde Latinoamérica y Caribe es parte de las iniciativas relacionadas al manifiesto "Poder, prestigio y valores olvidados: un manifiesto de estudios sobre desastres" (https://www.ipetitions.com/petition/power-prestigeforgotten-values-a-disaster), iniciado por académicos y activistas de estudios de desastres de todo el mundo. El manifiesto apunta a crear un futuro alternativo donde las epistemologías locales y los conocimientos de los pueblos oprimidos y olvidados puedan tener un espacio adecuado en los estudios de riesgos y desastres.

Este Número Especial de la Revista de Estudios Latinoamericanos sobre Reducción del Riesgo de Desastres (REDER) convocó a autores y autoras, que trabajan desde y sobre la región de Latinoamérica y Caribe, a presentar artículos que promuevan la pluralidad del conocimiento mediante la valoración de las ontologías y epistemologías locales, cuando corresponda, para descolonizar los estudios de desastres y trascender a los enfoques, fuentes, conceptos, metodologías, valores y lenguajes occidentales 'bien establecidos' que son predominantemente ajenos a los puntos críticos de desastres y riesgos que afectan a la región.

En la convocatoria de agosto de 2020 se preparó un listado de temas de interés que incluyeron: pensamiento social latinoamericano ante los desastres y epidemias; descolonización de la ciencia de los desastres; cosmovisiones, conocimiento de los pueblos oprimidos y saberes alternativos en la investigación de desastres; ecología de los saberes; antropología de los desastres; sociología de los desastres; nuevas geopolíticas y biopolíticas en los desastres y pandemias; modelos de desarrollo y producción social de los riesgos de desastres; procesos de vulneración, formas de opresión, desigualdad y pobreza en los desastres; racismo estructural y ambiental en los desastres; desastres olvidados y pueblos oprimidos; capitalismo del desastre; dimensiones del poder en la producción social de los riegos y en los desastres; éticas de investigación de desastres; perspectiva feminista de los estudios de desastres; movimientos sociales y estrategias de resistencia en los escenarios de riesgos y desastres; nuevos abordajes y métodos de investigación; y desafios de investigación en los enfoques inter y transdisciplinarios. Se recibieron 26 resúmenes en la fase inicial y 14 fueron seleccionados para su publicación.

Este Número Especial consta de dos partes, publicada una en julio de 2021 y otra en enero de 2022. Esta primera parte contiene ocho artículos de Brasil, Chile, Colombia, Ecuador, México y Puerto Rico. Se inicia con el artículo "Los mitos prehispánicos y los fenómenos naturales en Mesoamérica", de Joel F. Audefroy, para conocer los discursos y pensamientos de las culturas mesoamericanas - principalmente náhuatl y maya - relativos a los eventos desastrosos y cómo se integraron en sus prácticas religiosas. El texto examina cómo crearon mitos cosmogónicos alrededor del ciclo destrucción - renacimiento a partir de los eventos climáticos y geológicos, cuyas expresiones simbólicas perviven en la actualidad y se expresan en diversos rituales religiosos y tradiciones festivas.

El enfoque cultural también es abordado en el segundo artículo, titulado "Encuentros y desencuentros con la fórmula del riesgo y los desastres: tiempos para el cuidado y la reciprocidad". A partir de una reflexión teórica y metodológica desde una perspectiva crítica-feminista, María Teresa Armijos Burneo y Viviana Ramírez Loaiza, exponen los aprendizajes que construyeron con mujeres Embera pertenecientes al Cabildo urbano indígena Kurmadó (Risaralda, Colombia). Inspiradas por el pensamiento decolonial latinoamericano, las autoras proponen como pregunta de investigación: "¿qué pueden (o deben) aprender los estudios de riesgos y desastres de estas mujeres y sus saberes del habitar el territorio?" El artículo contiene una serie de reflexiones interesantes para el futuro de los estudios sobre la temática.

Las reflexiones sobre el proceso decolonial latinoamericano también se abordan en el tercer artículo, titulado "¿Descolonizar internamente? Rastreando los patrones militares y colonizadores en Ecuador a través de la gestión de riesgos", de autoría de Johannes M. Waldmüller y María Susana Robledo. Para los autores, en Ecuador predomina un enfoque técnico-científico y militar en la gestión de riesgos, basado en la seguridad nacional y la respuesta inmediata al desastre. En base a dos casos históricos, la erupción del volcán Tungurahua (1999-2006) y del terremoto costeño (2016-2018), los autores debaten la trayectoria colonial interna de la gestión de riesgos, subordinada a los intereses políticos y económicos de los gobiernos y élites de turno. Para Waldmüller y Robledo, si bien hubo ciertos cambios institucionales en la gestión del riesgo de 
desastres, aún prevalece un sesgo en la atención de los riesgos y desastres que le ha restado a las poblaciones locales, indígenas campesinas y afroecuatorianos, la oportunidad de empoderarse en la gestión de su propio territorio y sus recursos económicos asignados, prolongando formas del colonialismo interno, en clave étnica y de género.

La trayectoria colonial interna de la gestión de riesgos, subordinada a los intereses políticos y económicos de los gobiernos y élites de turno, también es analizada en el artículo "Gobierno del desastre en el neoliberalismo: una mirada genealógica desde los terremotos en Chile durante el siglo XX", de Juan Saavedra. A partir de fuentes diacrónicas, una serie de documentos históricos y artículos que refieren a los principales terremotos que afectan al territorio del país durante el período 1900-1980, el artículo interpreta las trazas históricas y discursivas que fundamentan el modo neoliberal de gobierno del desastre. Como resultado, se propone una interpretación de la trayectoria genealógica del gobierno neoliberal del desastre.

Las respuestas institucionales después de la emergencia se revisan también en el artículo "La vivienda ante desastres", de Aline Silveira Viana, de Brasil. Con el uso de la investigación documental y de entrevistas semiestructuradas, el artículo busca reflexionar sobre las respuestas institucionales y políticas habitacionales destinadas a la población afectada en los desastres. Entre los resultados, Aline Viana ha observado que la solución estandarizada de los conjuntos habitacionales verticales puede ir contra los anhelos y derechos de vivienda digna de los afectados, en especial, de las personas de edad avanzada en las zonas rurales. Para la autora, tales soluciones son reflejo de la manera de comprender el desastre y de los juegos de poder instalados en el territorio. Se concluye que, mientras los individuos y grupos no sean protagonistas en la decisión acerca del derecho a la vivienda digna, las políticas habitacionales en respuesta a los desastres seguirán perpetuando obstáculos y vulnerabilidades históricamente construidos.

No solo las memorias y narrativas de las personas de edad avanzada y los impactos en su derecho a la vivienda son importantes, sino también la creación de metodologías participativas que ayuden a escuchar aquellos que son invisibilizados. En el artículo "Gestión comunitaria del riesgo de desastre: una propuesta metodológica-reflexiva desde las metodologías participativas", de José Sandoval-Díaz y Soledad Martínez-Labrín, el lector es invitado a i) analizar el componente epistemológico de la investigación-intervención socio comunitaria, y ii) explorar la aplicabilidad de una propuesta de ocho saltos metodológicos participativos a la gestión local del riesgo. Los autores discuten la importancia (auto)reflexiva y metodológica-procedimental de incorporar estrategias participativas en propuestas investigativas-interventivas socio-comunitarias, como una contribución al agenciamiento y al mayor énfasis del conocimiento situado transdisciplinar, siendo un potencial vehículo movilizador de la reducción del riesgo de desastre a escala local desde las comunidades.

Las estrategias comunitarias también son abordadas en el artículo "Dignificando la Gestión de Riesgo de Desastres: liderazgos femeninos y estrategias comunitarias en el Campamento Dignidad, Santiago de Chile", de Valentina Acuña, Sofía Valdivieso y Leila Juzam.

Mediante un estudio de caso cualitativo y una aproximación que problematiza la escala local en asentamientos informales con múltiples amenazas -pandemia, riesgo de aluvión, inseguridad alimentaria, escasez hídrica, inundaciones e incendios-, se analizan las estrategias de organización comunitaria y redes de solidaridad externas e internas al territorio bajo liderazgos femeninos, relacionadas con la colaboración equitativa, apoyo voluntario, identificación política y transmisión de conocimientos locales. Los resultados sugieren dos grandes consideraciones respecto de la GRD en asentamientos informales. Primero, que el concepto de resiliencia en tanto adaptación, debe expandirse a partir de voces subalternas y la noción de dignidad como categoría moral para la transformación, dejando de lado ideas de estabilidad y despolitización. Y segundo, que esta debe estimular políticas de desarrollo que se hagan cargo del problema habitacional y desigualdades de género, problematizando las formas particulares de relación y organización y, por ende, las categorías tradicionales de hogar.

Las estrategias comunitarias y metodologías participativas pueden involucrar varios grupos sociales, incluyendo también a los jóvenes investigadores, que pueden ayudar en los procesos de reflexión sobre el pensamiento decolonial latinoamericano. En el artículo "¿Cómo, por quién y para qué? Investigación y labor creativa en el estudio de desastres en Puerto Rico", Tania López Marrero y Tamara Heartsill Scalley documentan en proceso de investigación-acción que resultó en el libro "Un cambio categoría 4: Memorias del huracán María", el cual incluye las narrativas 
personales de 15 estudiantes de pregrado de la Universidad de Puerto Rico. En el artículo, las autoras analizan el proceso de producción y diseminación del libro, como un mecanismo para afrontar las condiciones posteriores al desastre, además de aportar al desarrollo académico y profesional de los estudiantes. Las autoras reflejan cómo el libro contribuye a las aspiraciones del manifiesto 'Poder, prestigio y valores olvidados: un manifiesto de los estudios sobre desastres', el cual destaca la necesidad de producción de conocimiento por parte de aquellos que sufren los impactos de los desastres de manera que se reflejen adecuadamente las realidades locales en las que se desatan dichos desastres y que, además, redunden en beneficio a aquellos que viven en riesgo y experimentan estos procesos. En ese contexto, reflexionan y sugieren ciertos énfasis para adelantar los objetivos del manifiesto, de manera específica, y del estudio de desastres y reducción del riesgo, de manera más amplia.

Los artículos anteriores invitan a reflexionar sobre la importancia de tres elementos interrelacionados (Figura 1): por un lado, el conocimiento situado sobre los eventos climáticos y geológicos que tienen las poblaciones. Esto se refiere a cómo diferentes grupos y culturas crean y perciben las dinámicas naturales que ocurren en sus territorios y se expresan en prácticas, mitos, explicaciones, percepciones, actitudes y comportamientos socioculturales.

En segundo lugar, la gestión institucional para la prevención y manejo de desastres está supeditada a una perspectiva colonial, neoliberal y estructuralmente racista, que continúa excluyendo a grupos tradicionalmente marginados: afrodescendientes, indígenas, campesinos, jóvenes, personas mayores y mujeres. De esta manera, las respuestas institucionales, ya sea para la atención, manejo o prevención de los desastres, no son pertinentes culturalmente y perpetúan las vulnerabilidades de dichas poblaciones.

En tercer lugar, se pone énfasis en la urgencia de una perspectiva decolonial y feminista que incluya poblaciones excluidas, que permita negociaciones más equitativas en las relaciones de poder entre poblaciones e instituciones, que dé voz a las mujeres y otras minorías. En ese sentido, la gestión y reducción de riesgos y desastres requieren de políticas públicas más integrales, con un enfoque interseccional, de prevención y protección social, donde los protagonistas sean las familias y comunidades desde sus prioridades de atención diferenciadas.

\section{Políticas públicas}

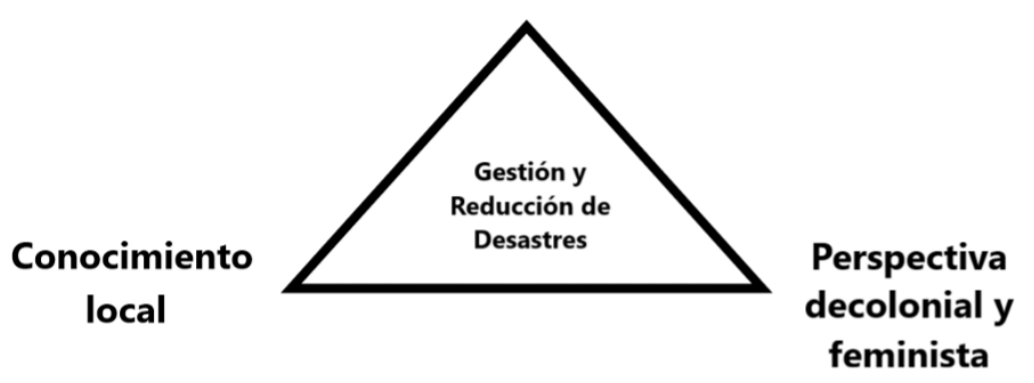

Figura 1. Tres elementos interrelacionados del proceso de descolonización de la ciencia de los desastres Fuente: Autores, 2021

En esta primera parte del número especial, se promueve un espacio para el debate en Latinoamérica y Caribe, involucrando a científicos y científicas que están trabajando en la temática. ¡Que disfruten de la lectura para fortalecer el pensamiento decolonial latinoamericano en los estudios de riesgos y desastres! En breve, la segunda parte de este número especial también estará disponible.

\section{EDITORES INVITADOS:}

Victor Marchezini, sociólogo, Brasil

Claudia González-Muzzio, arquitecta, Chile

Aracely Martínez Rodas, antropóloga, Guatemala 